\title{
Databases of proteins related to liquid-liquid phase separation
}

\author{
Qian $\mathrm{Li}^{1}$, Xi Wang ${ }^{2}$, Zhihui Dou ${ }^{2}$, Weishan Yang ${ }^{2}$, Beifang Huang ${ }^{2}$, Jizhong Lou ${ }^{3}$, and \\ Zhuqing Zhang ${ }^{1}$ \\ ${ }^{1}$ University of Chinese Academy of Sciences \\ ${ }^{2}$ University of Chinese Academy of Sciences \\ ${ }^{3}$ Institute of Biophysics Chinese Academy of Sciences
}

June 1, 2020

\begin{abstract}
Liquid-liquid phase separation (LLPS) of biomolecules has been investigated intensively in recent years, which underlies the formation of membrane-less organelles (MLOs) or biomolecular condensates. It contributes to the regulation of various physiological process and related disease development. Rapidly increasing number of studies have recently focused on the biological functions, driving and regulating mechanisms of LLPS in cell. Based on the mounting data generated in the investigations, six databases (LLPSDB, PhaSePro, PhaSepDB, DrLLPS, RNAgranuleDB, HUMAN CELL MAP) have been developed, designed directly based on LLPS studies or the components identification of MLOs. These resources are invaluable for deeper understanding of cellular function of biomolecular phase separation, as well as development of phase separating protein prediction and design. In this review, we summarize the data contents, annotations and organization for each of these databases, highlight their unique features, overlaps and fundamental differences, and further discuss their suitable applications.
\end{abstract}

\section{Hosted file}

Review_LLPS_Databases_202005_Proteins.pdf available at https://authorea.com/users/328647/ articles/455815-databases-of-proteins-related-to-liquid-liquid-phase-separation 